\title{
Seasonal Changes in Testes Vascularisation in the Domestic Cat (Felis domesticus): Evaluation of Microvasculature, Angiogenic Activity, and Endothelial Cell Expression
}

\author{
Graça Alexandre-Pires, ${ }^{1}$ Luísa Mateus, ${ }^{2}$ Catarina Martins, ${ }^{3}$ and Graça Ferreira-Dias ${ }^{1}$ \\ ${ }^{1}$ Morphology and Function Department, CIISA, Faculty of Veterinary Medicine of Lisbon, Technical University of Lisbon, \\ 1300-477 Lisbon, Portugal \\ ${ }^{2}$ Clinical Department, CIISA, Faculty of Veterinary Medicine of Lisbon, 1300-477 Lisbon, Portugal \\ ${ }^{3}$ Immunology Department, Faculty of Medical Sciences, 1169-056 Lisbon, Portugal
}

Correspondence should be addressed to Graça Alexandre-Pires, gpires@fmv.utl.pt

Received 31 July 2011; Accepted 25 October 2011

Academic Editor: Ruijin Huang

Copyright (C) 2012 Graça Alexandre-Pires et al. This is an open access article distributed under the Creative Commons Attribution License, which permits unrestricted use, distribution, and reproduction in any medium, provided the original work is properly cited.

Some male seasonal breeders undergo testicular growth and regression throughout the year. The objective of this study was to understand the effect of seasonality on: (i) microvasculature of cat testes; (ii) angiogenic activity in testicular tissue in vitro; and (iii) testicular endothelial cells expression throughout the year. Testicular vascular areas increased in March and April, June and July, being the highest in November and December. Testes tissue differently stimulated in vitro angiogenic activity, according to seasonality, being more evident in February, and November and December. Even though CD143 expression was higher in December, smaller peaks were present in April and July. As changes in angiogenesis may play a role on testes vascular growth and regression during the breeding and non-breeding seasons, data suggest that testicular vascularisation in cats is increased in three photoperiod windows of time, November/December, March/April and June/July. This increase in testicular vascularisation might be related to higher seasonal sexual activity in cats, which is in agreement with the fact that most queens give birth at the beginning of the year, between May and July, and in September.

\section{Introduction}

The study of the vasculature of the testis has attracted scientists' attention for many centuries, especially at the end of the 19th and throughout the 20th century. This research has been performed in a large variety of animal species such as rat, mouse, rabbit, guinea pig, dog, ram, bull, boar, horse, marsupials, man, and other primates [1-4]. It has been long known that the physiologic role of the pampiniform plexus, on thermoregulation of the testes. This is a highly efficient countercurrent heat exchanger in which the arterial blood is precooled before it reaches the testis, while venous blood is warmed to body temperature before it returns to the abdomen [5]. It is nevertheless very rare to find references on the vasculature of cat testes (Felis domesticus).

Angiogenesis is physiologically modulated through a dynamic balance between the production and release of angi- ogenic/mitogenic growth factors or antiangiogenic/anti-mitogenic substances [6-9]. In the adult, physiological angiogenesis is mostly restricted to the female reproductive tract during the ovarian/uterine cycle [10-13]. Nevertheless, in the male, physiologic gonadal angiogenesis has also been described. Testicular angiogenesis is known to increase during testicular recrudescence in seasonal breeders such as the hamster [14] or to decrease in response to food restriction in rabbits [15].

Testis mass and sperm production vary throughout the year in animals such as the bonnet monkey or the European hamster [16-18]. It is also known that sexual activity is influenced by geographic location $[19,20]$. Seasonality of domestic feral cats in the northern hemisphere is referred to occur throughout a wide range of months, when day light is prolonged for 12 hours or longer [21]. Testicular growth and regression occur throughout the year with simultaneous 


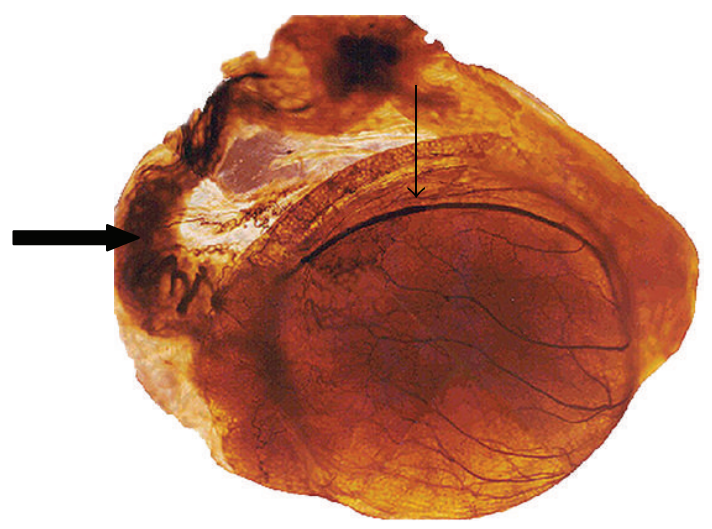

(a)

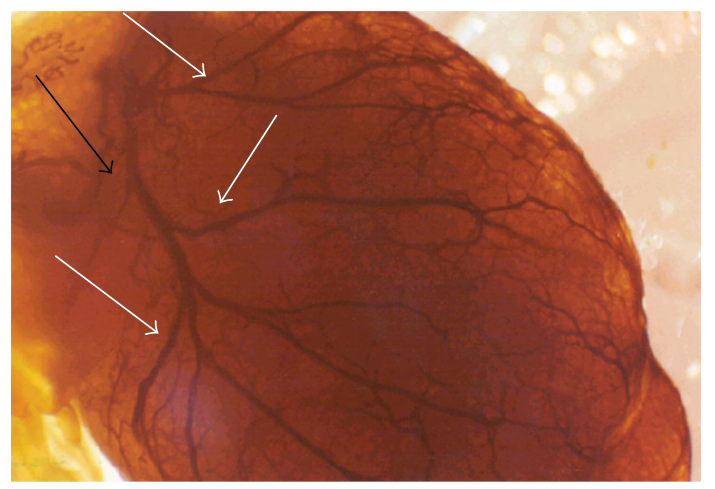

(b)

FIgURE 1: ( $\mathrm{a}$ and $\mathrm{b}$ ) Injection-diaphanisation technique: a single testicular artery, slightly convoluted in the spermatic cord (thick arrow). This vessel winds around its caudal pole to reach the ventral border (thin arrow), where it starts to branch in a monopodical way, giving away branches to both sides of the testis (white arrows).

fast changes in blood flow that appears to be stimulated by angiogenic substances and inhibited by antiangiogenic factors [22-25]. The objective of this study was to understand the effect of seasonality on (i) microvasculature of cat testes; (ii) angiogenic activity in testicular tissue in vitro; (iii) testicular endothelial cells expression throughout the year (January-December).

\section{Material and Methods}

2.1. Sample Collection. A total of 516 testes from adult domestic stray cats $(n=378)$ were collected and used for the present work. Testes were obtained from cats of indeterminate breed, weight, and age that underwent euthanasia, at the Official Kennel of Lisbon, Portugal (latitude $38^{\circ} 43^{\prime} \mathrm{N}$ ). This organization is recognized by the European Convention for the Protection of Small Animals.

Experiments were monitored by competent veterinary authorities and approved by the ethical committee of the Faculty of Veterinary Medicine (Lisbon, Portugal). Authors G. Alexandre-Pires and L. Mateus, are holders of an FELASA (Federation of European Laboratory Animal Science Associations) grade $\mathrm{C}$ certificate, which allows designing and conducting laboratory animal experimentation in the European Union.

2.2. Injection-Diaphanisation Technique. Soon after euthanasia, 10 testis were collected from adult cats $(n=5)$ in order to be used for injection-diaphanisation technique. injection-diaphaniszation or clearing technique was performed as follows: (i) washing of the vascular bed with distilled water: (ii) injection of $10 \%$ jelly and a colloidal suspension of barium sulphate (Micropaque-Laboratório Guerbet, Portugal), in a $25 \%$ dilution, at $37^{\circ} \mathrm{C}$; (iii) fixation phase which was performed by using $10 \%$ formaldehyde solution; (iv) bleaching phase, that was accomplished by immersion of testis in hydrogen peroxide for 30 minutes. (v) "Freeze substitution method" was performed, which consists in freezing the organ to $-20^{\circ} \mathrm{C}$ and damping it

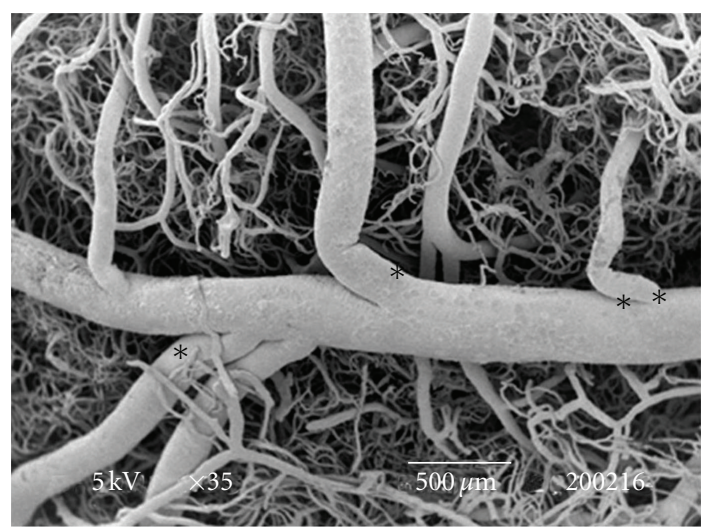

FIGURE 2: SEM: micrograph of cat testicular artery that gives away branches to both sides of the testis. These vessels are commonly the 1st- $\left.{ }^{*}\right)$ and 2 nd-order arteries $(* *)$. Bar $=500 \mu \mathrm{m}$.

in pure commercial acetone at $-20^{\circ} \mathrm{C}$ (Maialab, Portugal). (vi) Clarification phase was followed, which consists of the immersion of testis' slices in Spalteholz liquid, composed of benzyl benzoate and methyl salicilate (ChemNet Europe). Finally, samples were observed by transillumination (Wild, M3Z, Switzerland).

2.3. Scanning Electron Microscopy. Scanning electron microscopy (SEM) was performed on microvascular corrosion casts of cat testicles $(n=10)$ and intact testicular tissue $(n=5)$. SEM technique for microvascular corrosion casts was performed according to the following protocol: immediately after euthanasia a polyethylene catheter was introduced in the thoracic aorta of the cat. Postmortem fixation was achieved by injection of $2.2 \%$ glutaraldehyde in phosphate buffer solution ( $\mathrm{pH}$ 7.28) inside the vascular bed. After, an acrylic resin known as "Mercox" (Vilene Hospital, Tokyo, Japan) was injected through the catheter. After polymerization of the resin the corrosion was carried out through the use of a $24 \% \mathrm{NaOH}$ solution (SIGMA, Portugal). Testis corrosion casts were mounted on stubs, coated with gold 


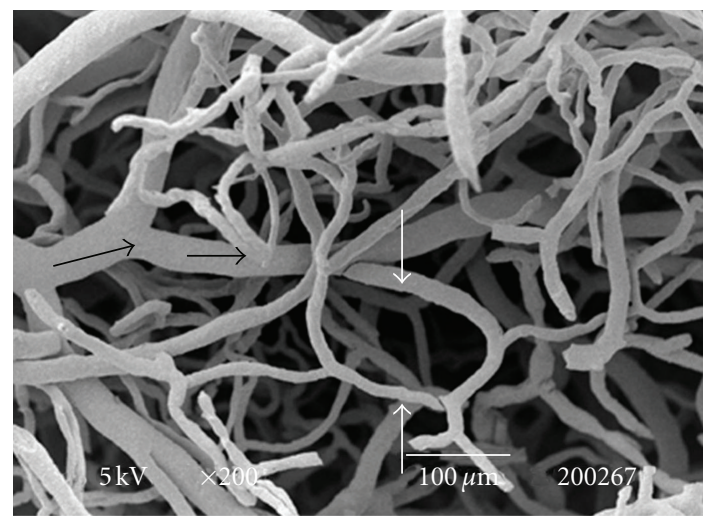

(a)

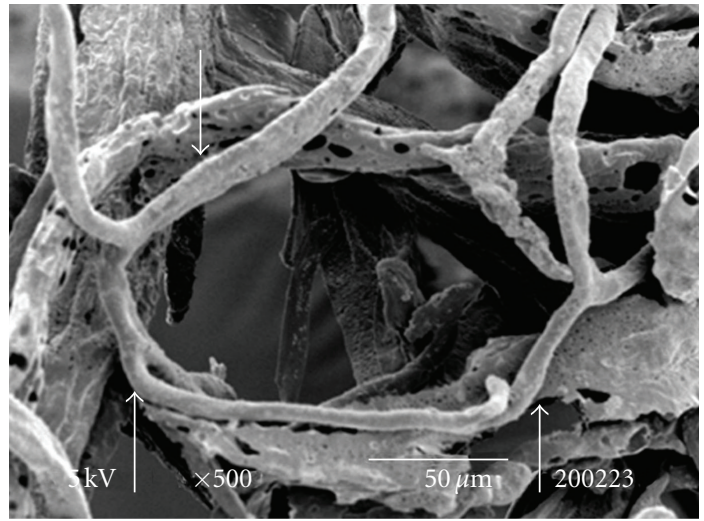

(b)

FIgURE 3: (a) SEM: dichotomic branches from different pre-capillary arterioles (black arrow) show peritubular patterns determined by the layout of the seminiferous tubules (white arrow). Bar $=100 \mu \mathrm{m}$. (b) SEM: Idem. Peritubular vascularisation (arrows). Bar $=50 \mu \mathrm{m}$.

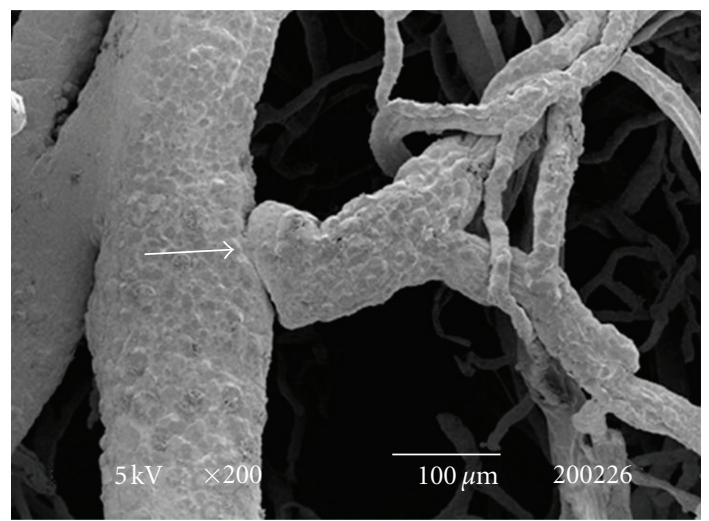

Figure 4: SEM: an ostial valvulae (arrow) can be observed in a 2 nd order artery. Bar $=100 \mu \mathrm{m}$.

palladium and observed in a scanning electronic microscope (JEOL-JSM-5410).

Scanning electronic microscopy technique for intact tissue was performed following the next steps: cat testes were immersed in Karnovsky's solution (Sigma-Aldrich, Portugal). Afterwards they were rinsed in cacodylate buffer and post-fixed in a $2 \%$ osmium tetroxide solution for 1 hour. The pieces were rinsed once again with cacodylate buffer and subsequently dehydrated in a graded ethanol series. Samples were dried using the critical point drying method and coated with gold salts. The same electronic microscope (JEOL-JSM5410) was used to study and photograph these structures.

2.4. Flow Cytometry Analysis. For one year, a total of 236 cat testes were collected monthly immediately after euthanasia $(n=118)$. Testes were removed with a surgical blade and collected into a sterile tube with $1 \mathrm{~mL}$ of RPMI 1640 (GibcoBrl, USA). After disaggregation of the tissue with a surgical blade samples that corresponded to the total amount of testis mass were centrifuged at $190 \times \mathrm{g}$ for $10 \mathrm{~min}$ and adjusted to a concentration of $10^{6}$ cells per $100 \mu \mathrm{L}$ in phosphatebuffered saline solution (PBS). Aliquots of $10^{6}$ cells were

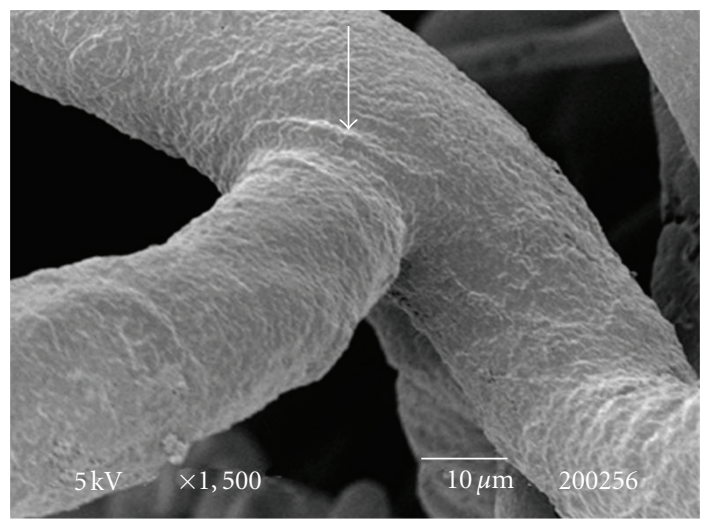

FIGURE 5: SEM: intra-arterial cushions are observed in pre-capillary arterioles (arrow). Bar $=10 \mu \mathrm{m}$.

incubated for $30 \mathrm{~min}$ at $4^{\circ} \mathrm{C}$ with $10 \mu \mathrm{L}$ of CD143 mouse anti-human antibody (MCA2057 from AbdSerotec, $170 \mathrm{KD}$ somatic form), which recognizes angiotensin converting enzyme mainly in endothelial cells. Red blood cells were lysed with BD FACS Lysing Solution (BD Biosciences, San Jose, CA, USA) for 10 minutes. After centrifugation, at $190 \mathrm{~g}$ for 10 minutes, the pellet was resuspended in $500 \mu \mathrm{L}$ of BD FACS Flow (BD Biosciences, San Jose, CA, USA). Fluorochromeconjugated secondary antibodies, namely Goat anti-mouse IgG : RPE, (from AbDSerotec, UK) were added, vortexed and incubated for 15-30 minutes. Finally, to wash off antibody excess following staining, $1.5 \mathrm{~mL}$ of PBS was added to each tube. Cell acquisition was performed on a BD FACS Calibur flow cytometer (BD Biosciences, San Jose, CA, USA) and data were analyzed using Paint-A-Gate Pro and Cell-Quest Pro software (BD Biosciences, San Jose, CA, USA).

2.5. Microvascular Density Evaluation. Monthly and throughout a whole year, testes were collected after euthanasia from domestic stray cats, for histology and for tissue culture ( $n=120$, from the total number of animals) were collected. After removal of the epididymis, testes were cut 


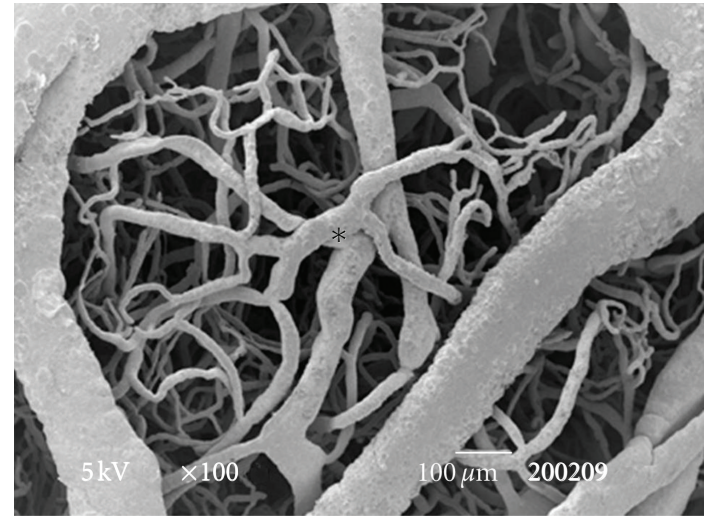

(a)

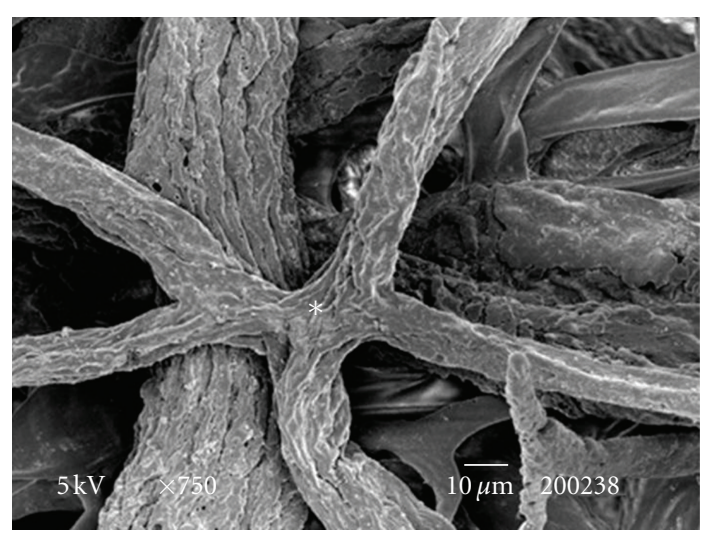

(b)

FIGURE 6: (a) SEM: peritubular vascularisation. Terminal arterioles often branch into vessels located at different segmental levels. This fact originates stelatae images $(*)$. Bar $=100 \mu \mathrm{m}$. (b) SEM: the same aspect. Bar $=10 \mu \mathrm{m}$.

transversally for different assays such as the determination of vascular areas and mitogenesis assays.

For histological studies, samples were placed in $4 \%$ buffered formaldehyde, fixed overnight, and dehydrated in a series of ethanol solutions and embedded in paraffin. Sections were cut with a rotary microtome (Leica Microsystems Nussloch GmbH, Nussloch, Germany), stained with Periodic Schiff reagent (Sigma), and evaluated under a light microscope (Olympus Ck40, Wetzlar-Nauborn, Germany). For each testis, 28 randomly selected fields were photographed using a light microscope at 1000x magnification. Testicular vascular areas were measured by using computerized image analysis (Scion Image, NIH, USA). Vascular density (arterioles, venules, and capillaries) was assessed as the percentage of the area occupied by blood vessels with respect to the entire area of each micrograph $[12,15,26]$. For each animal/testis, the vascular area was considered as the mean value of the area of blood vessels for each animal on the same 28 randomly selected fields.

2.6. Mitogenesis Assays. For mitogenesis assays, $60 \mathrm{mg}$ of testicular tissue (10 cats from each month) were incubated in $2 \mathrm{~mL}$ culture medium, for 18 hours, in a tissue incubator (Biosafe Eco-Integra Biosciences, Chur, Switzerland) at $37^{\circ} \mathrm{C}$, $5 \% \mathrm{CO}_{2}, 95 \%$ air, on a shaker (Titertek, Huntsville, $\mathrm{AL}$, USA) AT 1500 RPM. Culture medium consisted of DMEM and Ham's F12 (1:1 V/V) supplemented with $0.1 \mathrm{BSA}$, penicillin $(100 \mathrm{IU} / \mathrm{mL})$, and streptomycin $(100 \mu \mathrm{g} / \mathrm{mL})$, being all reagents from Sigma. After incubation, media were stored at $-70^{\circ} \mathrm{C}$ for further mitogenesis assays. Negative controls consisted of culture medium alone.

The ability of media conditioned by testicular tissue to stimulate angiogenic activity was indirectly assessed by mitogenesis/proliferation of Bovine Aortic Endothelial Cells (BAEC; kindly donated by Dr. Dale A. Redmer, North Dakota State University, Fargo, ND, USA), as referred by Redmer et al., [27].

Briefly, BAEC cells $\left(2 \times 10^{4}\right.$ cells $\left./ \mathrm{mL}\right)$ were allowed to attach to the bottom of 24-well culture plate (NucleonNunc, Ballerup, Denmark) for 24 hours, in a tissue incubator

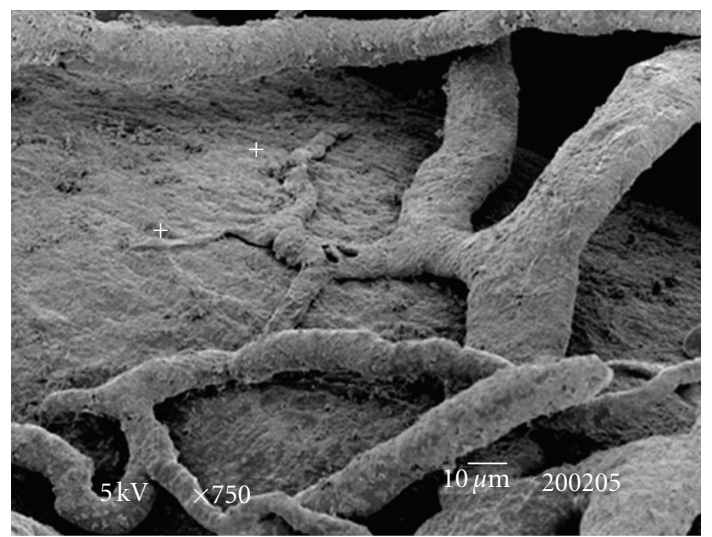

FIGURE 7: SEM: vasa vasorum $(+)$ over a 2nd-order arteriola. Bar $=$ $10 \mu \mathrm{m}$.

(Biosafe Eco-Integra Biosciences) at $37^{\circ} \mathrm{C}, 5 \% \mathrm{CO}_{2}, 95 \%$ air [12]. Samples of testicular conditioned media (or controls) were added in triplicate wells at a final concentration of $30 \%$ (30\% conditioned media $+70 \%$ DMEM) and incubated for 72 hours. Vascular endothelial growth factor (VEGF; Sigma) was used as a positive control, at different concentrations $(10,20,25$ and $50 \mathrm{ng} / \mathrm{mL})$ to evaluate the ability of an angiogenic factor to stimulate BAEC proliferation under the same experimental conditions [12]. In order to assess BAEC proliferative response, the number of these cells in each well was determined using a Neubauer chamber under the light microscope (Olympus CK40) and further compared with positive controls (VEGF) and negative controls (no tissue added). The percentage of BAEC proliferation in media conditioned by testes was calculated with respect to negative controls. Cell proliferation or mitogenesis in response to negative control culture media was considered $100 \%$.

2.7. Statistical Analysis. Statistical significance among data regarding microvascular areas and angiogenic activity in cat testes throughout the year were analysed by one-way ANOVA. The level of significance was set at $P<0.05$. 


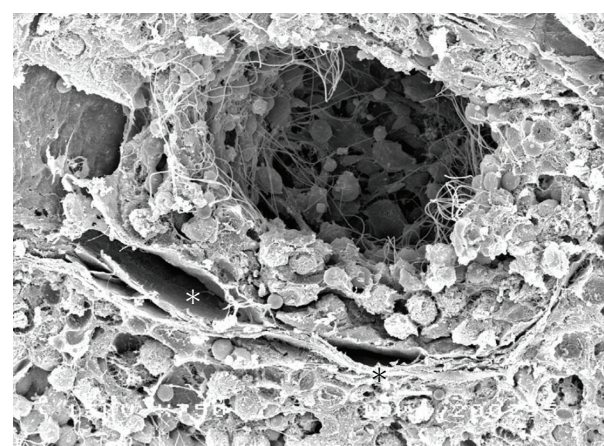

(a)

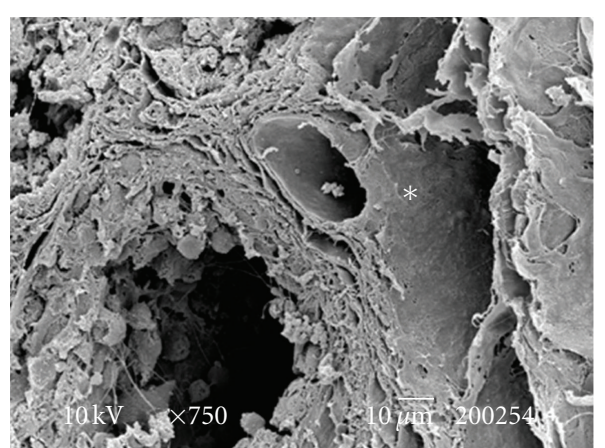

(b)

Figure 8: (a, b) SEM over intact tissue: seminiferous tubules showing different stages of maturation. Vessels $(*)$ can be seen surrounding these tubules. Bar $=10 \mu \mathrm{m}$.

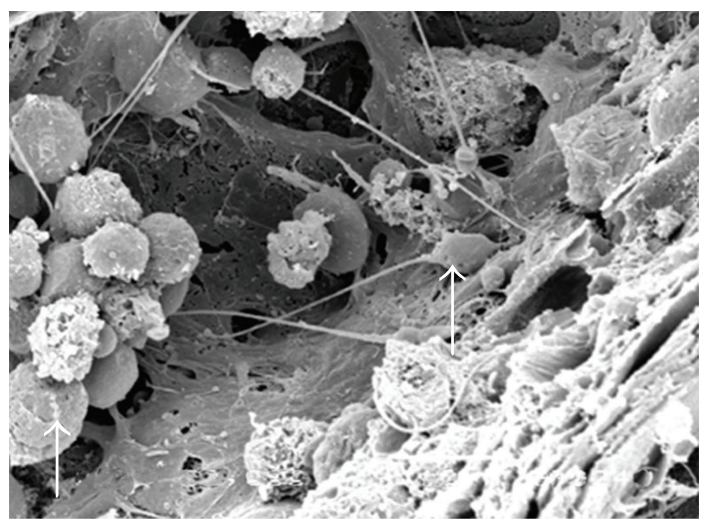

FIGURE 9: SEM over intact tissue: spermatogonias at different stages of maturation (arrows) inside a seminiferous tubule. Bar $=10 \mu \mathrm{m}$.

Whenever a significant difference was observed, a Bonferroni multiple-comparison test was performed.

\section{Results}

3.1. Testicular Microvascularization. The testes microvascularization was classified on the basis of the classification adopted and applied to the placenta and to the dog's spleen and third eyelid [28-30]. The present study has shown that cat testis is supplied by a single testicular artery, slightly convoluted in the spermatic cord, where it is surrounded by largely anastomosed veins of the very rich pampiniform plexus (Figure 1(a)). Upon reaching the hilum of the testis, the testicular artery winds around its caudal pole to reach the ventral border, where it starts to branch in a monopodical way, giving away branches to both sides of the testis (Figures 1(b) and 2). These branches, usually small calibre arteries or 1st-order arterioles, course undivided for a variable length under the tunica albuginea and then sink into the testicular parenchyma where they branch, originating successively 1st-order $(200-100 \mu \mathrm{m}), 2$ nd-order $(100-30 \mu \mathrm{m})$, and precapillary or terminal arterioles $(30-10 \mu \mathrm{m})$. Although first divisions of the testicular artery are given in a monopodical way, branches located distally from the artery originate

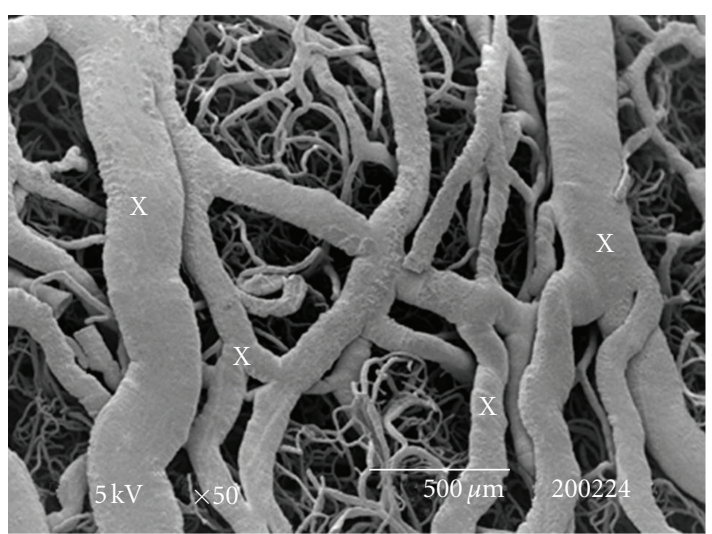

FIGURE 10: SEM: anastomosed network of flattened veins $(\mathrm{X})$. Bar $=$ $500 \mu \mathrm{m}$.

branches in a dichotomic way and the same pattern can be seen inside the testicular parenchyma (Figure 3(a)). The microvascular distribution pattern in the cat testes appears to be largely determined by the organ histology and especially the layout of the seminiferous tubules; therefore, 2nd-order arterioles, pre-capillary, as well as the capillaries $(10-4 \mu \mathrm{m})$, and postcapillary venules $(10-30 \mu \mathrm{m})$ that follow have almost a well defined hexagonal pattern of vessels surrounding the intertubular and peritubular patterns seminiferous tubules (Figure 3(b), 8(a), and 8(b)). Inside seminiferous tubule one can observe spermatogonia cells at different stages of maturation (Figure 9). Vascular features important in redirecting the blood flow and the amount of flow can be also observed in testis microvascularization, such as ostial valvulae (Figure 4) and intraarterial cushions (Figure 5). Vasa vasorum can be also observed (Figure 7).

Terminal arterioles often branch into vessels located at different segmental levels giving rise to an anastomotic net pattern (Figure 6(a)). As monopodical ramifications and dichotomic ramifications can be seen sprouting at the top of these vessels, one can observe stelatae ramifications (Figure $6(\mathrm{~b}))$.

2nd-order (30-100 microns) and 1st-order (100-200 microns) venules drain predominantly into an important 


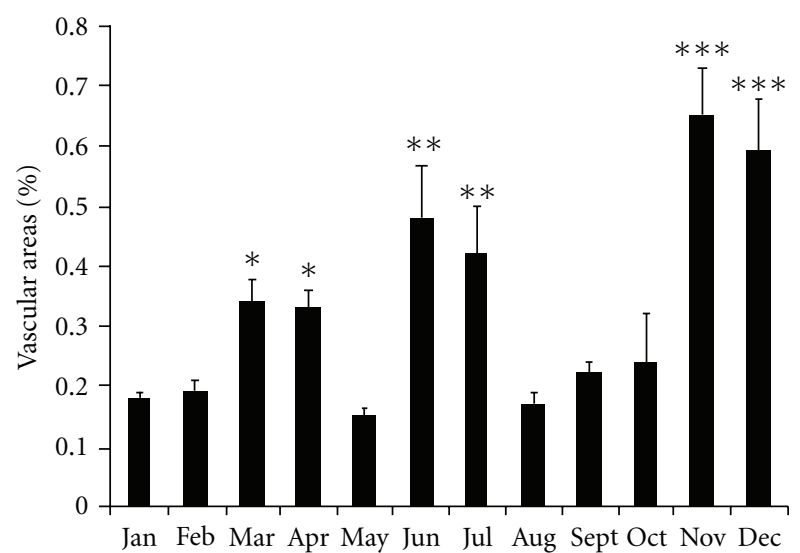

FIgURE 11: Vascular areas in testes throughout the year. A significant increase was observed in three different times of the year. A higher increase was observed in November and December when compared to the other months $\left({ }^{* * *} P<0.001\right)$. Different symbols show significant differences: $\left({ }^{* *} P<0.01 ;{ }^{*} P<0.05\right)$.

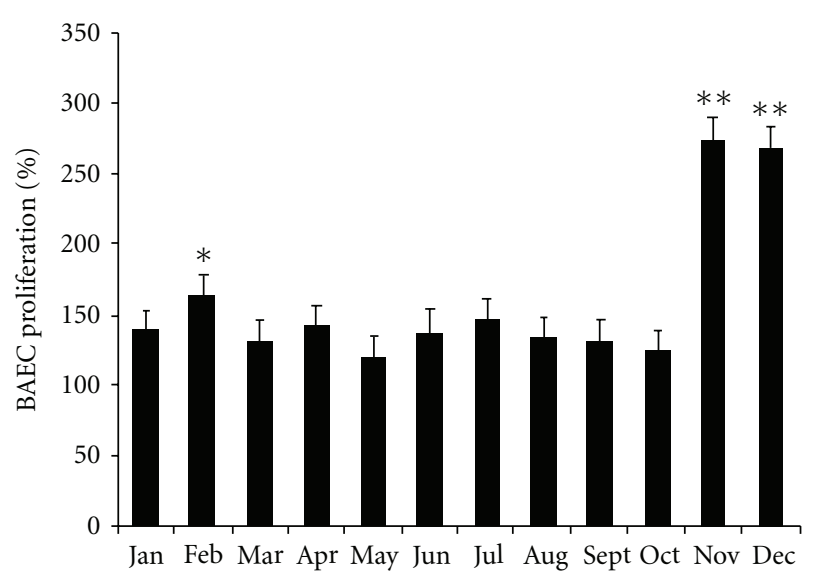

FIGURE 12: BAEC mitogenesis capacity of conditioned media by testes collected throughout the year. Different symbols show significant differences with other months $\left({ }^{*} P<0.05\right.$; $\left.{ }^{*} P<0.001\right)$.

anastomosed network of flattened veins, laid out in two superimposed subalbuginea vascular layers, which ultimately collect into a cranial plexus that form the pampiniform plexus (Figure 10).

Our data points out an increase in microvascular areas in three different times of the year: March and April $(P<$ $0.05)$; June and July $(P<0.01)$; November and December $(P<0.001)$, when compared to other months. In fact, it was in November and December that cat testes presented the highest microvascular areas when compared to the remaining months (Figure 11).

It was also observed that cat testes tissue stimulates in vitro angiogenic activity differently. All testicular tissue showed the capability to increase BAEC proliferation, when compared to negative control throughout the study. In addition, there was an increase in BAEC mitogenic activity in February $(P<0.05)$ and November and December $(P<$ 0.001 ), when compared to the other months (Figure 12).

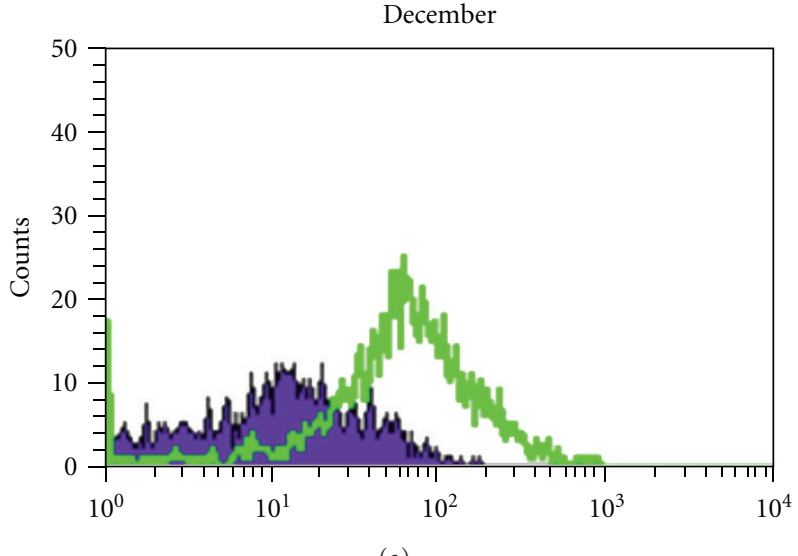

(a)

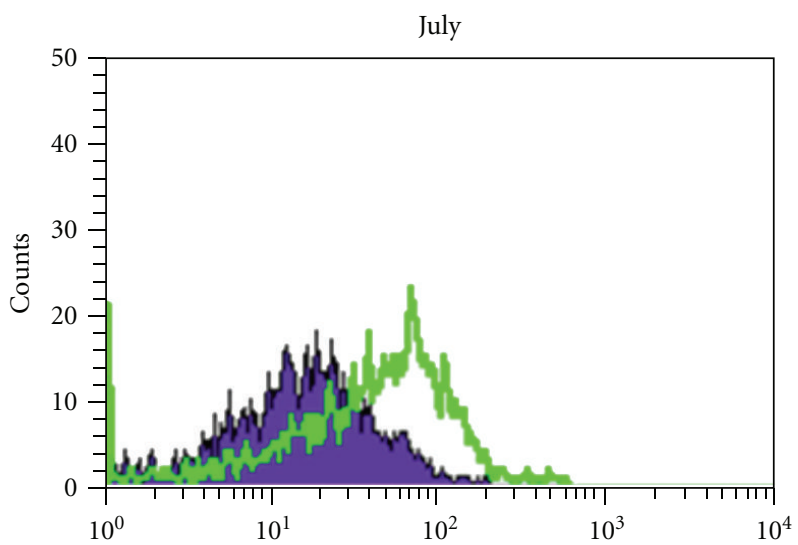

(b)

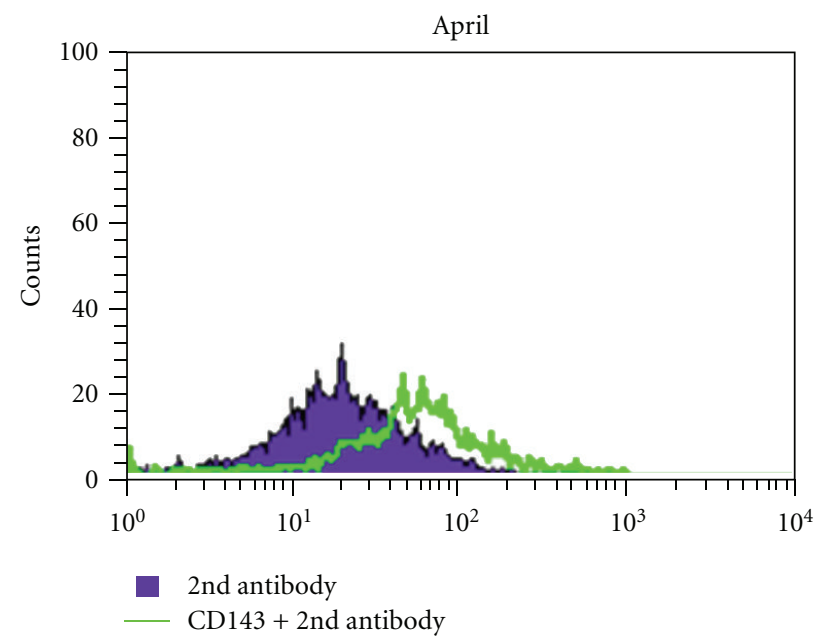

(c)

FIGURE 13: Examples of histograms showing the expression of CD143 in testes in different months of the year.

Considering flow cytometry studies regarding testicular endothelial cell evaluation, it was observed a 20 to $30 \%$ increase in CD143 expression in December $(P<0.001)$, although smaller peaks were present also in April and July $(P<0.01)$. The major immunophenotypic features are illustrated in Figures 13-14. 


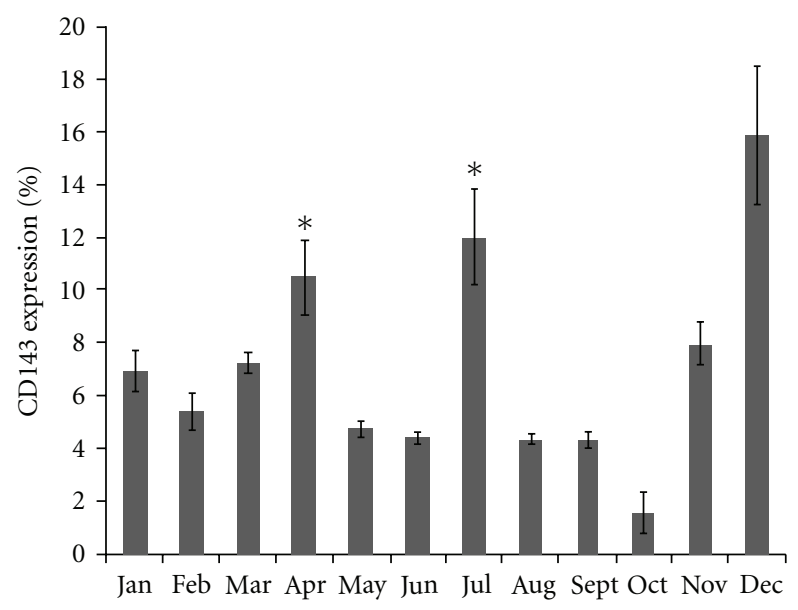

Figure 14: CD143 expression in the supernatant of ground testes along an entire year. An increase in CD143 expression was observed in December $(P<0.001)$ although smaller peaks were also observed in April and July $(P<0.01)$.

\section{Discussion}

The formation of new blood vessels from a preexisting vasculature, known as angiogenesis, is a quite infrequent process in adult mammals as a physiologic process. Both angiogenic and antiangiogenic factors play a regulatory role on angiogenesis [7, 31]. In the female reproductive tract during the ovarian/uterine cycle it occurs as physiologic changes [10-13], as well as in the male reproductive tract during gonadal recrudescence in seasonal breeders $[14,24$, $32,33]$.

It is known that testicular arteries of many animals (humans, mice, and rats) run from the abdominal aorta to the testes showing various configurations (straight, spiral, meandering, or coiled forms). Each species exhibits a specific pattern that may play several roles in protection of normal spermatogenesis, such as allowing wide mobility of the testes on physical attack, heat loss throughout the pampiniform plexus [34].

When evaluating the microvascular arrangement of arterial and venous vessels of cat testes, our data highlight the presence of intertubular and peritubular patterns found in precapillary or terminal arterioles, as well as in capillaries and postcapillary venules which seem to be similar to the patterns found in other species $[35,36]$, being the angioarchitecture largely determined by the testicular histology and especially the layout of the seminiferous tubules. Besides, venous organization in the cat testes might contribute for an efficient reduction in blood temperature within the testis, as secondand first-order venules which drain predominantly into an anastomosed network of flattened veins, laid out in two superimposed subalbuginea vascular layers that ultimately collect into a cranial plexus that form the pampiniform plexus. This outstanding venous plexiform apparatus, covering the cranial pole of the gonad seems to be particularly well suited to perform an important role in the thermoregulatory mechanisms of the testis, as mentioned before for bull [37], dolphin [38], and human [39].
Seasonal reproduction is mainly under photoperiodic control and is common among mammals at all latitudes. Photoperiod (day length) is a cue for temporal information in order to initiate and terminate seasonally appropriate morphological, physiological, and behavioural modifications that maximize survival as well as reproductive success [24]. This tool allows organisms to essentially track time-of-year and to anticipate relatively predictable annual variations in important environmental parameters [40, 41]. Photoperiod information obtained by the retina is transduced into a physiological signal via the pineal hormone, which secretes melatonin [40]. The molecular mechanisms at the hypothalamo-hypophysial level are involved in the secretion of melatonin at night which subsequently influences the gonadal axis in mammals [42]. Variations in response to photoperiod are seen not only among species but also between breeding populations within a species and between individuals within single-breeding populations [40]. This appears to be the result of differences in responsiveness to photoperiod or melatonin target sites responsiveness [40]. The molecular mechanisms that regulate vascular development and regression in response to environmental status are a subject with a lot of gaps in our knowledge. Seasonal changes in testicular capillary blood flow [43] and volume and density of testicular microvasculature [14] have been reported in several species that breed seasonally. It has also been pointed out that regression and regrowth of the whitefooted mouse (Peromyscus leucopus) testes positively correlates with vascular endothelial growth factor (VEGF) protein expression [23]. Nevertheless, controversial studies in the White-Footed mouse do not support the idea that melatonin helps to regulate seasonal reproduction by acting in the testes to inhibit steroidogenesis [44]. Genetic differences have been pointed out in some species on both the degree and duration of reproductive quiescence [45]. Our data show that cat testicular tissue collected from different periods of the year was able to show angiogenic activity. The fact that the highest peak of BAEC mitogenesis in the testes occurred in November and December, and the greatest testicular vascular area was found in November and December as well, clearly points out that at this time of the year physiologic events are involved in the vascular growth of testes. Besides, in the same photoperiod window, a significant increase in CD143 expression in cat testes, which corresponds to a rise in endothelial cell proliferation (20 to $30 \%$ of total cell number), also occurred in December, which may indicate a stimulation in angiogenesis/vascular proliferation. This increase in angiogenic activity might be ascribed to testes increase in angiogenic/mitogenic factors, such as vascular endothelial growth factor (VEGF), angiopoietins (Angs), basic fibroblast growth factor (bFGF), and epidermal growth factor (EGF), considered to be crucial for new blood vessel growth [46-49]. Nevertheles, this raise in angiogenic activity in cats testes in Nov/Dec months might not only be due to increased angiogenic factors, but also to a reduction in the production of antiangiogenic factors, such as angiostatin, endostatin $[8,50]$, thrombospondins [51], and platelet factor 4- PF-4 [52]. In fact, an increase in VEGF expression in rat testes stimulated vascular endothelial cells and germ cells 
proliferation [53], while its inhibition was responsible for a reduction on in vitro testes vascular density [54]. Besides, gonadotrophins might also act directly on testis endothelial cells as tissue-specific angiogenic factors by modulating a more favorable vascular supply [55]. Since VEGF has been shown to stimulate testosterone release by rat Leydig cells in a dose dependent fashion [56], and testicular germ cell survival and sperm production in bulls [57], the present increase in vascularization might be related to an increase in testosterone secretion needed for cat reproduction function.

Research models that account for photoperiodic time measurement by a circadian mechanism postulate that the timing of light exposure, rather than the total amount of light, is critical to the pineal gland perception of day length and that might explain sudden modifications, with gaps of few months in testes activity. The present study points out three peaks considering vascular areas, namely, March and April, June and July, and November and December. Crossing this information with results obtained on mitogenesis assays and CD143 expression, it can be observed that December is committed to testes changes as it was demonstrated an increase in BAEC mitogenesis and an increase in CD143 expression also in this month.

Although a clear time-window can be related to November and December, a similar pattern can be observed in March-April and June-July, although with lower peaks. The increase in BAEC mitogenesis in February might represent an intermediate physiological state such as the ones observed between peripubertal and active adult testes [58].

Angiogenesis modifications might relate to function since testicular vasculature is unique in several ways. This is due to the presence of unfenestrated endothelial cells in the testis, which show about 10-fold higher proliferation rate when compared to other organs [59]. Endothelial cell mitogenesis, blood flow, and vascular permeability in testis could be further increased by gonadotrophin stimulation of Leydig cells [59-62]. As changes in angiogenesis may play a role on vascular growth and regression of the testes during the breeding and nonbreeding season in the male cat respectively, altogether, these data suggest that testicular vascularisation in cats appears to be predominantly increased in three photoperiod windows of time, November/December, March/April, and June/July. These findings are in accordance with the fact that most queens give birth at the beginning of the year, between May and July and in September (65-67 days of gestation). These windows of seasonality might be the response of circadian oscillators to the timing of light exposure, rather than the total amount of light. This mechanism might be involved in changes in testicular vascular pattern in the domestic cats that might influence their reproductive performance in the northern hemisphere at this range of latitude $\left(38^{\circ} 43^{\prime} \mathrm{N}\right)$.

\section{Acknowledgments}

The authors would like to thank Dr. Dale A Redmer, from the Department of Animal Sciences, North Dakota State University, Fargo, ND, USA, for kindly donating them bovine aortic endothelial cells (BAECs) isolated in his laboratory. Authors also thank Mrs. Glória Nunes for technical assistance. This work was supported by Interdisciplinary Centre of Research in Animal Health (CIISA), Faculty of Veterinary Medicine of Lisbon, Technical University of Lisbon, Portugal.

\section{References}

[1] S. Ergun, J. Stingl, and A. F. Holstein, "Microvasculature of the human testis in correlation to Leydig cells and seminiferous tubules," Andrologia, vol. 26, no. 5, pp. 255-262, 1994.

[2] S. A. Gunn and T. C. Gould, "Vasculature of the testes and adnexa," in Handbook of Physiology, R. O. Greep and E. B. Astwood, Eds., pp. 117-172, William and Wilkins, Baltimore, Md, USA, 1975.

[3] C. Oliveira, A. M. Orsi, C. A. Vicentini, and H. E. Duarte, "Microvascularização testicular no hamster (Mesocricetus auratus)," Naturalia, vol. 16, pp. 103-109, 1991.

[4] T. R. Weerasooriya and T. Yamamoto, "Three-dimensional organization of the vasculature of the rat spermatic cord and testis. A scanning electron-microscopic study of vascular corrosion casts," Cell and Tissue Research, vol. 241, no. 2, pp. 317323, 1985.

[5] B. G. Brackett, "Male reproduction in mammals," in Dukes' Physiology of Domestic Animals, O. William, Ed., pp. 670-691, 12th edition, 2004.

[6] O. Hudlicka, "Development of microcirculation: capillary growth and adaptation," in Handbook of Physiology, E. M. Renkin and C. C. Michael, Eds., vol. 4 of Section 2: The Cardiovascular System, Part 1: Microcirculation, pp. 165-216, American Physiological Society, Washington, DC, USA, 1 edition, 1984.

[7] J. Folkman and M. Klagsbrun, "Angiogenic factors," Science, vol. 235, no. 4787, pp. 442-447, 1987.

[8] M. C. Espinosa Cervantes and A. Rosado García, "Angiogenesis in reproductive physiology. Follicular development, formation and maintenance of the corpus luteum," Ginecologia $y$ Obstetricia de Mexico, vol. 70, pp. 17-27, 2002.

[9] T. M. Hazzard, R. M. Rohan, T. A. Molskness, J. W. Fanton, R. J. D'Amato, and R. L. Stouffer, "Injection of antiangiogenic agents into the macaque preovulatory follicle: disruption of corpus luteum development and function," Endocrine, vol. 17, no. 3, pp. 199-206, 2002.

[10] L. P. Reynolds, S. D. Killilea, and D. A. Redmer, "Angiogenesis in the female reproductive system," FASEB Journal, vol. 6, no. 3, pp. 886-892, 1992.

[11] H. G. Augustin, K. Braun, I. Telemenakis, U. Modlich, and W. Kuhn, "Ovarian angiogenesis: phenotypic characterization of endothelial cells in a physiological model of blood vessel growth and regression," American Journal of Pathology, vol. 147, no. 2, pp. 339-351, 1995.

[12] G. Ferreira-Dias, P. P. Bravo, L. Mateus, D. A. Redmer, and J. A. Medeiros, "Microvascularization and angiogenic activity of equine corpora lutea throughout the estrous cycle," Domestic Animal Endocrinology, vol. 30, no. 4, pp. 247-259, 2006.

[13] R. P. Roberto da Costa, G. Ferreira-Dias, L. Mateus et al., "Endometrial nitric oxid production and nitric oxid synthasis in the equine endometrium: relationship with microvascular density during the estruos cycle," Domestic Animal Endocrinology, vol. 32, pp. 287-302, 2007.

[14] A. Mayerhofer, A. P. Sinha Hikim, A. Bartke, and L. D. Russell, "Changes in the testicular microvasculature during photoperiod-related seasonal transition from reproductive 
quiescence to reproductive activity in the adult golden hamster," Anatomical Record, vol. 224, no. 4, pp. 495-507, 1989.

[15] M. Carvalho, L. Mateus, F. Afonso et al., "Testicular angiogenic activity in response to food restriction in rabbits," Reproduction, vol. 137, no. 3, pp. 509-515, 2009.

[16] R. Medhamurthy, R. Suresh, S. S. Paul, and N. R. Moudgal, "Correlation of seasonal changes in sperm output with endocrinological changes in the adult male bonnet monkey, Macaca radiata," Journal of Biosciences, vol. 19, no. 1, pp. 67-74, 1994.

[17] R. B. Moreland, M. Elaine Richardson, N. Lamberski, and J. A. Long, "Characterizing the reproductive physiology of the male Southern black howler monkey, Alouatta caraya," Journal of Andrology, vol. 22, no. 3, pp. 395-403, 2001.

[18] S. Monecke, M. Saboureau, A. Malan, D. Bonn, M. MassonPévet, and P. Pévet, "Circannual phase response curves to short and long photoperiod in the European Hamster," Journal of Biological Rhythms, vol. 24, no. 5, pp. 413-426, 2009.

[19] B. Pérez and E. Mateos, "Effect of photoperiod on semen production and quality in bucks of Verata and Malaguena breeds," Small Ruminant Research, vol. 23, no. 1, pp. 23-28, 1996.

[20] S. M. Hammoudi, A. Aït-Amrane, T. B. Belhamiti, B. Khiati, A. Niar, and D. Guetarni, "Seasonal variations of sexual activity of local bucks in western Algeria," African Journal of Biotechnology, vol. 9, no. 3, pp. 362-368, 2010.

[21] M. J. Daniels, T. C. M. Wright, K. P. Bland, and A. C. Kitchener, "Seasonality and reproduction in wild-living cats in Scotland," Acta Theriologica, vol. 47, no. 1, pp. 73-84, 2002.

[22] F. H. Bronson and P. D. Heideman, "Seasonal regulation of reproduction in mammals," in Physiology of Reproduction, E. Knobil and J. D. Neill, Eds., pp. 541-583, Raven, New York, NY, USA, 1994.

[23] K. A. Young and R. J. Nelson, "Short photoperiods reduce vascular endothelial growth factor in the testes of Peromyscus leucopus," American Journal of Physiology, vol. 279, no. 3, pp. R1132-R1137, 2000.

[24] B. J. Prendergast, L. J. Kriegsfeld, and R. J. Nelson, “Photoperiodic polyphenisms in rodents: neuroendocrine mechanisms, costs, and functions," Quarterly Review of Biology, vol. 76, no. 3, pp. 293-325, 2001.

[25] L. M. Pyter, A. K. Hotchkiss, and R. J. Nelson, "Photoperiodinduced differential expression of angiogenesis genes in testes of adult Peromyscus leucopus," Reproduction, vol. 129, no. 2, pp. 201-209, 2005.

[26] G. M. Ferreira-Dias, P. M. Serrão, J. F. Costa Durão, and J. R. Silva, "Microvascular development and growth of uterine tissue during the estrous cycle in mares," American Journal of Veterinary Research, vol. 62, no. 4, pp. 526-530, 2001.

[27] D. A. Redmer, A. T. Grazul, J. D. Kirsch, and L. P. Reynolds, "Angiogenic activity of bovine corpora lutea at several stages of luteal development," Journal of Reproduction and Fertility, vol. 82, no. 2, pp. 627-634, 1988.

[28] G. Alexandre-Pires, "Aspects of the placental vascularisation of the rabbit female (Oryctolagus cuniculus) when gestation occurs under induced anaemic conditions," Brazilian Journal of Morphology Science, vol. 15, pp. 85-92, 1998.

[29] G. Alexandre-Pires, D. Pais, and J. A. Esperança Pina, "Intermediary spleen microvasculature in Canis familiarismorphological evidences of a closed circulation," Anatomia, Histologia, Embryologia, vol. 32, pp. 1-8, 2003.

[30] G. Alexandre-Pires, M. C. Algueró, L. Mendes-Jorge, H. Trindade, M. Correia, and J. A. Esperança Pina, "Immunophenotyping of lymphocyte subsets in the third eyelid tissue in dogs (Canis familiaris): morphological, microvascular, and secretory aspects of this ocular adnexa," Microscopy Research and Technique, vol. 71, no. 7, pp. 521-528, 2008.

[31] M. Klagsbrun and P. A. D'Amore, "Regulators of angiogenesis," Annual Review of Physiology, vol. 53, pp. 217-239, 1991.

[32] J. K. Findlay, "Angiogenesis in reproductive tissues," Journal of Endocrinology, vol. 111, no. 3, pp. 357-366, 1986.

[33] R. J. Tomanek and G. C. Schatteman, "Angiogenesis: new insights and therapeutic potential," Anatomical Record, vol. 261, no. 3, pp. 126-135, 2000.

[34] H. Terayama, M. Naito, Y. Nakamura et al., "Corrosion casts of convoluted testicular arteries in mice and rats," Systems Biology in Reproductive Medicine, vol. 51, no. 6, pp. 471-480, 2005.

[35] J. E. Anderson and J. A. Thliveris, "Testicular histology in streptozotocin-induced diabetes," Anatomical Record, vol. 214, no. 4, pp. 378-382, 1986.

[36] M. Kormano, "Microvascular supply of the regenerated rat testis following cadmium injury," Virchows Archiv Abteilung A Pathologische Anatomie, vol. 349, no. 3, pp. 229-235, 1970.

[37] L. F. C. Brito, A. E. D. F. Silva, R. T. Barbosa, and J. P. Kastelic, "Testicular thermoregulation in Bos indicus, crossbred and Bos taurus bulls: relationship with scrotal, testicular vascular cone and testicular morphology, and effects on semen quality and sperm production," Theriogenology, vol. 61, no. 2-3, pp. 511-528, 2004.

[38] S. A. Rommel, D. A. Pabst, W. A. McLellan, J. G. Mead, and C. W. Potter, "Anatomical evidence for a countercurrent heat exchanger associated with dolphin testes," Anatomical Record, vol. 232, no. 1, pp. 150-156, 1992.

[39] A. I. Sealfon and A. W. Zorgniotti, "A theoretical model for testis thermoregulation," Advances in Experimental Medicine and Biology, vol. 286, pp. 123-135, 1991.

[40] B. D. Goldman, "Mammalian photoperiodic system: formal properties and neuroendocrine mechanisms of photoperiodic time measurement," Journal of Biological Rhythms, vol. 16, no. 4, pp. 283-301, 2001.

[41] M. J. Paul, I. Zucker, and W. J. Schwartz, "Tracking the seasons: the internal calendars of vertebrates," Philosophical Transactions of the Royal Society B, vol. 363, no. 1490, pp. 341361, 2008.

[42] S. Yasuo and T. Yoshimura, "Comparative analysis of the molecular basis of photoperiodic signal transduction in vertebrates," Integrative and Comparative Biology, vol. 49, no. 5, pp. 507-518, 2009.

[43] J. Joffre and M. Joffre, "Seasonal changes in the testicular blood flow of seasonally breeding mammals: dormouse, Glis glis, ferret, Mustella furo, and fox, Vulpes vulpes," Journal of Reproduction and Fertility, vol. 34, no. 2, pp. 227-233, 1973.

[44] L. K. Knotts, B. C. Bruot, and J. D. Glass, "Melatonin does not affect in vitro secretion of testosterone in white-footed mouse testis," Journal of Pineal Research, vol. 5, no. 6, pp. 521-526, 1988.

[45] W. D. Gram, H. W. Heath, H. A. Wichman, and G. R. Lynch, "Geographic variation in peromyscus leucopus: short-day induced reproductive regression and spontaneous recrudescence," Biology of Reproduction, vol. 27, no. 2, pp. 369-373, 1982.

[46] D. Gospodarowicz, J. Cheng, and G. M. Lui, "Corpus luteum angiogenic factor is related to fibroblast growth factor," Endocrinology, vol. 117, no. 6, pp. 2383-2391, 1985.

[47] R. L. Stouffer, J. C. Martínez-Chequer, T. A. Molskness, F. $\mathrm{Xu}$, and T. M. Hazzard, "Regulation and action of angiogenic factors in the primate ovary," Archives of Medical Research, vol. 32, no. 6, pp. 567-575, 2001. 
[48] T. Shimizu, J. Y. Jiang, H. Sasada, and E. Sato, "Changes of messenger RNA expression of angiogenic factors and related receptors during follicular development in gilts," Biology of Reproduction, vol. 67, no. 6, pp. 1846-1852, 2002.

[49] D. J. Hicklin and L. M. Ellis, "Role of the vascular endothelial growth factor pathway in tumor growth and angiogenesis," Journal of Clinical Oncology, vol. 23, no. 5, pp. 1011-1027, 2005.

[50] P. C. Maisonpierre, C. Suri, P. F. Jones et al., "Angiopoietin-2, a natural antagonist for Tie2 that disrupts in vivo angiogenesis," Science, vol. 277, no. 5322, pp. 55-60, 1997.

[51] C. Tamanini and M. De Ambrogi, "Angiogenesis in developing follicle and corpus luteum," Reproduction in Domestic Animals, vol. 39, no. 4, pp. 206-216, 2004.

[52] C. Perollet, Z. C. Han, C. Savona, J. P. Caen, and A. Bikfalvi, "Platelet factor 4 modulates fibroblast growth factor 2 (FGF2) activity and inhibits FGF-2 dimerization," Blood, vol. 91, no. 9, pp. 3289-3299, 1998.

[53] H. Hashimoto, T. Ishikawa, K. Yamaguchi, M. Shiotani, and M. Fujisawa, "Experimental ischaemia-reperfusion injury induces vascular endothelial growth factor expression in the rat testis," Andrologia, vol. 41, no. 4, pp. 216-221, 2009.

[54] R. C. Bott, R. M. McFee, D. T. Clopton, C. Toombs, and A. S. Cupp, "Vascular endothelial growth factor and kinase domain region receptor are involved in both seminiferous cord formation and vascular development during testis morphogenesis in the rat," Biology of Reproduction, vol. 75, no. 1, pp. 56-67, 2006.

[55] K. Reisinger, N. Baal, T. McKinnon, K. Münstedt, and M. Zygmunt, "The gonadotropins: tissue-specific angiogenic factors?" Molecular and Cellular Endocrinology, vol. 269, no. 1-2, pp. 65-80, 2007.

[56] G. S. Hwang, S. W. Wang, W. M. Tseng, C. H. Yu, and P. S. Wang, "Effect of hypoxia on the release of vascular endothelial growth factor and testosterone in mouse TM3 Leydig cells," American Journal of Physiology, vol. 292, pp. E1763-E1769, 2007.

[57] K. C. Caires, J. De Avila, and D. J. McLean, "Vascular endothelial growth factor regulates germ cell survival during establishment of spermatogenesis in the bovine testis," Reproduction, vol. 138, no. 4, pp. 667-677, 2009.

[58] M. B. Frungieri, S. I. Gonzalez-Calvar, A. Bartke, and R. S. Calandra, "Influence of age and photoperiod on steroidogenic function of the testis in the golden hamster," International Journal of Andrology, vol. 22, no. 4, pp. 243-252, 1999.

[59] O. Collin and A. Bergh, "Leydig cells secrete factors which increase vascular permeability and endothelial proliferation," International Journal of Andrology, vol. 19, no. 4, pp. 221-228, 1996.

[60] B. P. Setchell and R. M. Sharpe, "Effect of injected human chorionic gonadotrophin on capillary permeability, extracellular fluid volume and the flow of lymph and blood in the testes of rats," Journal of Endocrinology, vol. 91, no. 2, pp. 245254, 1981.

[61] B. Geesaman, J. Villanueva-Meyer, D. Bluestein, L. Miller, I. Mena, and J. Rajfer, "Effects of multiple injections of HCG on testis blood flow," Urology, vol. 40, no. 1, pp. 81-83, 1992.

[62] A. Bergh, J. E. Damber, and N. Van Rooijen, "The human chorionic gonadotrophin-induced inflammation-like response is enhanced in macrophage-depleted rat testes," Journal of Endocrinology, vol. 136, no. 3, pp. 415-419, 1993. 

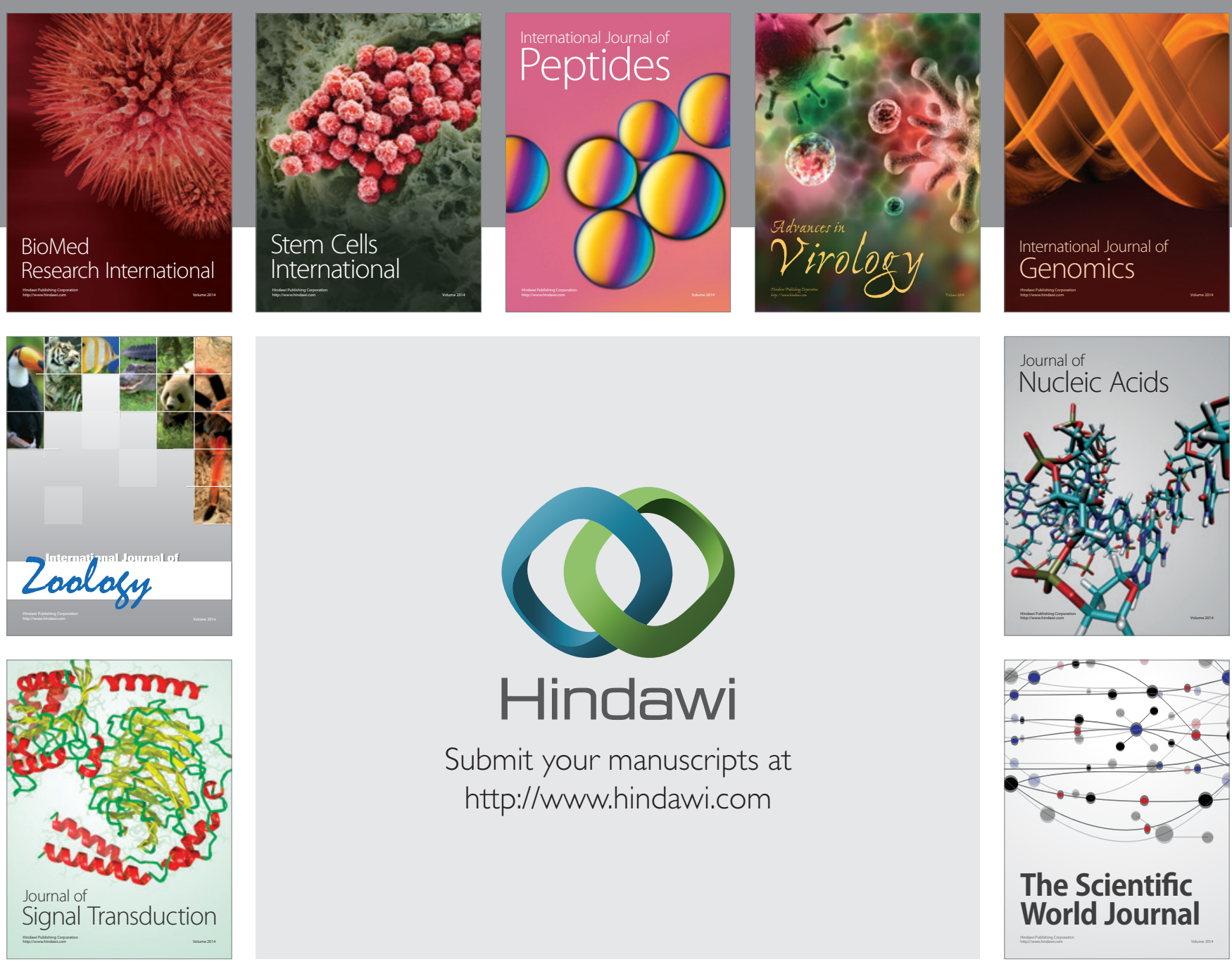

Submit your manuscripts at

http://www.hindawi.com
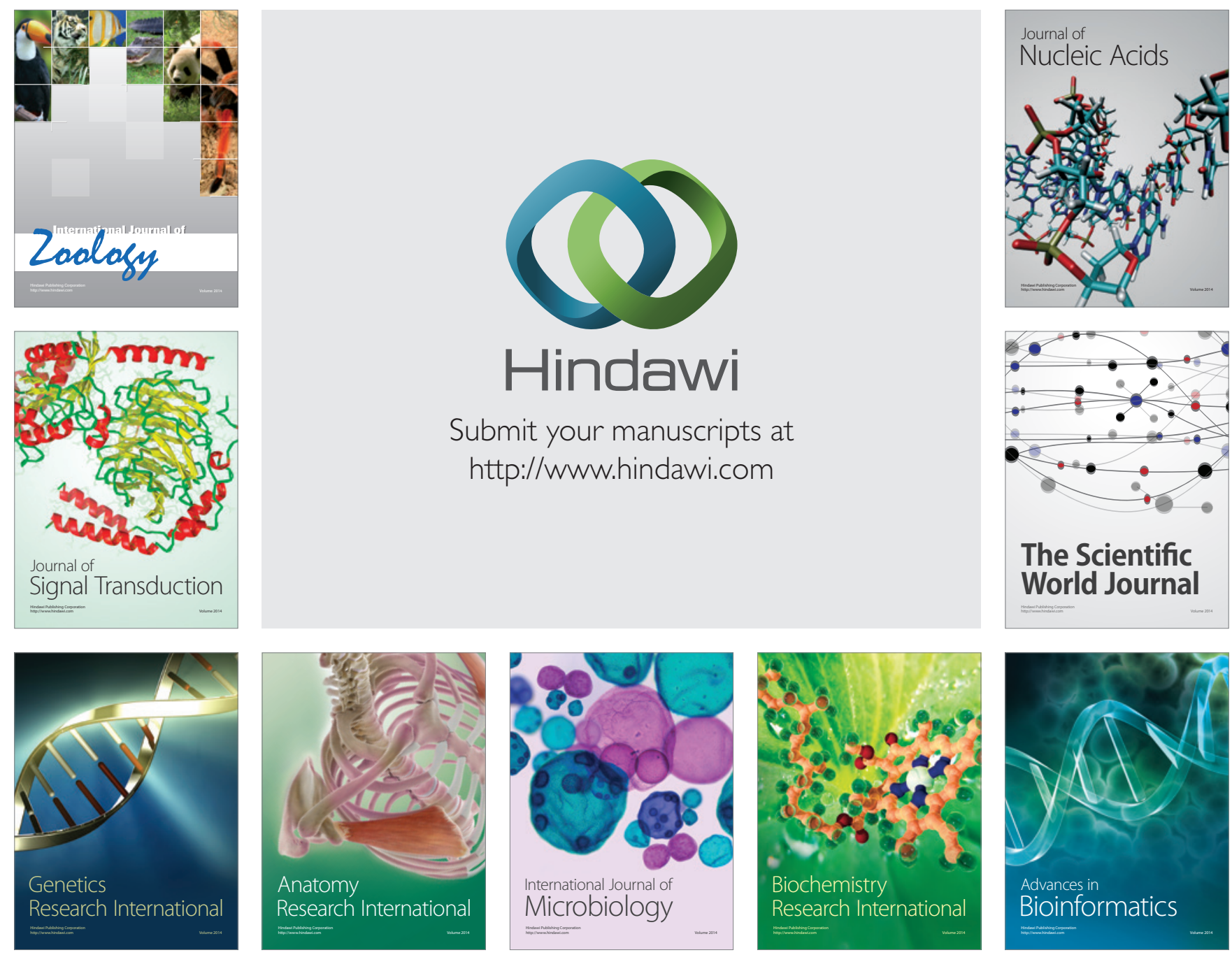

The Scientific World Journal
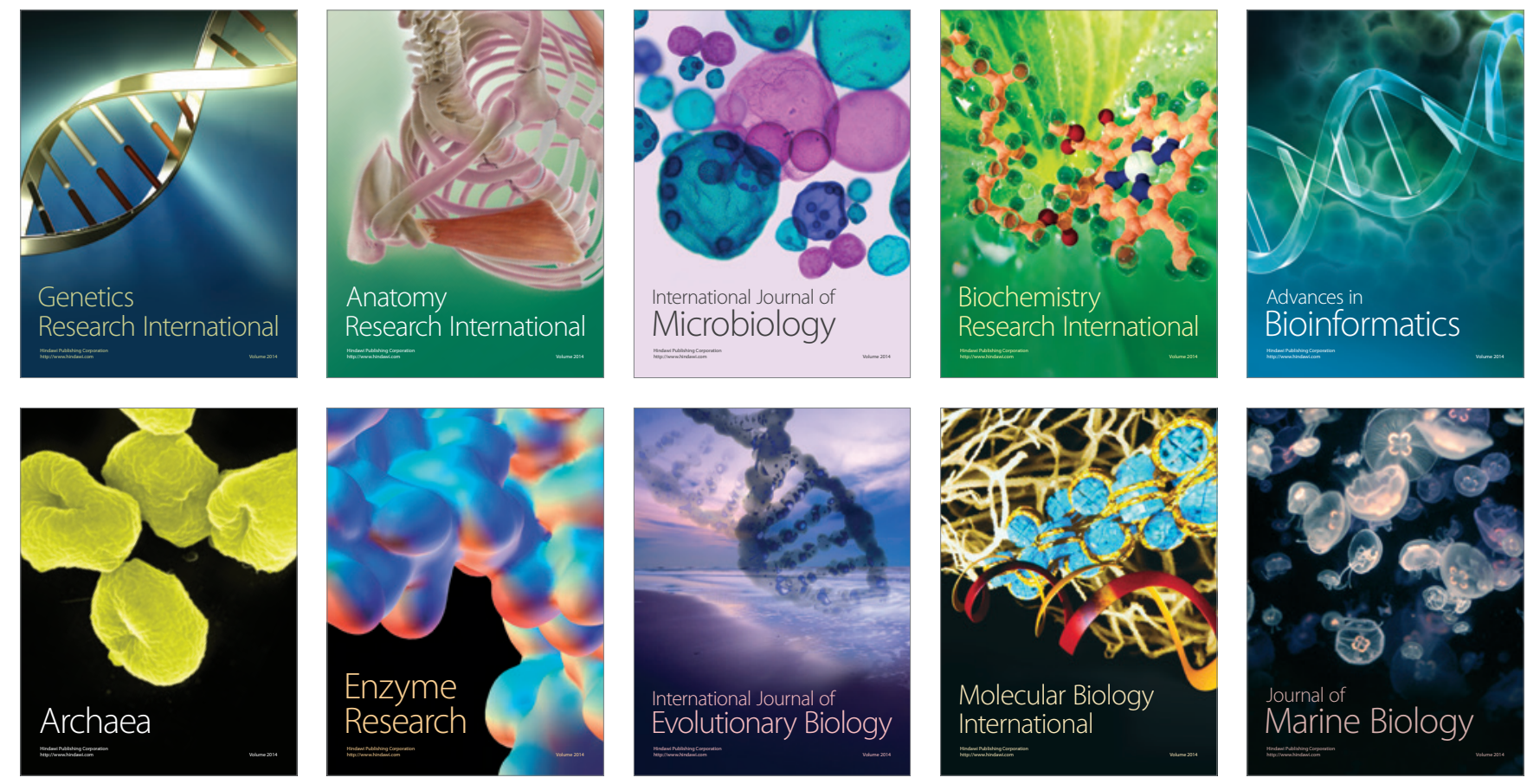